\title{
Informe: infecciones por Clostridium difficile en el Hospital de Urgencia Asistencia Pública (HUAP) Dr. Alejandro del Río de Santiago, Chile
}

\author{
Sociedad Chilena de Infectología a solicitud del Ministerio de Salud de Chile
}

\author{
Report: Clostridium difficile outbreak at the Dr. Alejandro del Río Emergency \\ Hospital in Santiago, Chile
}

\section{Contenidos}

Introducción

Metodología de trabajo

531

Descripción del HUAP

Epidemiología de las infecciones por

C. difficile en el HUAP ................................ 533

Factores potenciales involucrados ................. 533

Factores del hospedero o paciente ........... 534

Factores ambientales-asistenciales ........... 534

Factores del agente ................................. 535

Medidas implementadas .............................. $\quad 536$

Impacto de las medidas implementadas ........ 537

Recomendaciones 537

Comentarios finales
- Notificaciones y control de medidas generales, aislamiento de cohorte y contacto.

- Verificación de existencia de normas y protocolos de uso de antimicrobianos en unidades de atención clínica

- Análisis de laboratorio clínico del punto de vista microbiológico en el HUAP.

- Análisis del consumo global de antimicrobianos.

SOCHINF agradece la confianza depositada por el Ministerio de Salud y la oportunidad de colaborar en el análisis y control de esta situación, contribuyendo así a mejorar la calidad y seguridad de atención de los pacientes. Asimismo, agradece la excelente atención que recibió la comisión visitante en el HUAP por los equipos y personas entrevistadas, quienes entendieron que los resultados de este trabajo servirán para mejorar la atención de los pacientes con los que ellos trabajan cada día.

\section{Metodología de trabajo}

El análisis de la situación fue realizado mediante reuniones y entrevistas en terreno entre los días 23 y 30 de abril de 2012. Se realizaron reuniones con el Director Médico (Dr. Emilio Villalón), Sub-Directora Médica (Dra. Patricia Méndez) y Sub-Directora de Enfermería (EU. $\mathrm{M}^{\mathrm{a}}$ Angélica Arancibia), con la Unidad de Infecciones Asociadas a Atención de Salud (Dr. Claudio Vargas, EU. Carolina Herrera y EU. Ana María Seguel) y con los jefes del Servicio de Medicina (Dr. Patricio González), Cirugía (Dra. Ana María Pacheco) y UCI (Dr. Enzo Sáez). Además se visitó la sección de Bacteriología del Laboratorio Central de HUAP y la Unidad de Farmacia. Asimismo, se obtuvo copia de múltiples documentos incluyendo:

- Análisis de gestión clínica asociada a la producción hospitalaria (HUAP) 2011.

- Acreditación de HUAP en IIH 2007.

- Notificación de brotes efectuada por Unidad de IAAS al SEREMI (febrero-marzo 2012). 
- Infección por $C$. difficile en el HUAP: el brote de julio 2011-marzo 2012 en contexto.

- Norma de uso racional de antimicrobianos HUAP vigente (revisada el 2009).

- Memorandum que indica el uso de metronidazol para cobertura de anaerobios en neumonía aspirativa en el Servicio de Urgencia HUAP y que restringe el uso de clindamicina y levofloxacina (20 de abril 2012).

- Contrato con empresa de aseo y bases técnicas (septiembre 2011).

\section{Resultados}

Los resultados de la evaluación presentan en forma consecutiva:

- Descripción del HUAP.

- Situación epidemiológica de las infecciones por $C$. difficile.

- Factores involucrados asociados al paciente, ambientales-asistenciales y microbiológicos.

- Medidas tomadas y su impacto.

\section{Descripción del HUAP}

Características generales del HUAP. Consta de 293 camas, de las cuales 30 corresponden a UCI e Intermedio y otras 12 a la Unidad de Quemados con 8 camas de intensivo y 4 de intermedio (14,3\% de las camas destinadas a pacientes críticos o de intermedio). Aunque la edad promedio de los pacientes atendidos en el HUAP es de 56 años con un rango de 14 a 107 años, el 47,3\% de los ingresos tiene $\geq 60$ años. El índice de complejidad de pacientes atendidos en el HUAP es mayor que el promedio nacional y eso significa que los pacientes que ingresan tienen en términos comparativos, patologías más complejas, requieren más recursos por intensidad diagnóstica y terapéutica asociada. Ello queda reflejado en el hecho que $50 \%$ de los pacientes que egresan del HUAP han tenido una condición grave o moderada y que $14 \%$ de los egresos requirió en algún momento camas críticas o de intermedio versus $6,4 \%$ de los egresos nacionales. En el año 2011 se registraron 10.778 egresos hospitalarios.

Infraestructura. Las unidades de hospitalización generales cuentan con salas de 8 camas y con un baño con 2 o 3 WC cada 40 pacientes aproximadamente. En las salas con 8 camas no existe separación espacial de un metro entre pacientes o unidad clínica. Se ha logrado, ya por varios años, no tener pacientes hospitalizados en camillas en los pasillos del hospital. Sin embargo, ante la incapacidad de absorber toda la demanda de hospitalización, existe cada día, entre 28 y 36 pacientes hospitalizados en el Servicio de Urgencia en espera de traslado.
Laboratorio de Microbiología. Depende del Laboratorio Central y cuenta con personal estable de dos profesionales tecnólogos médicos más un tecnólogo y un ayudante técnico en cuarto turno. No existe personal médico con horas asignadas al laboratorio ni hay participación de personal del laboratorio en la Unidad de Infecciones Asociadas a la Atención de Salud (IAAS). Cuenta con capacidad de procesar la mayoría de las muestras microbiológicas habituales. No se realizan en este laboratorio técnicas de diagnóstico viral (por ejemplo virus respiratorios) ni estudio de tuberculosis; estas últimas se derivan a otro hospital. Están disponibles técnicas para el diagnóstico de IAAS aunque en algunos casos con restricciones a horario hábil (ej. cultivo cuantitativo de aspirado endotraqueal). No se describe suspensión de exámenes por falta de insumos. Existe disponibilidad de hemocultivos automatizados sin restricciones de acceso y desde marzo de 2011 se cuenta con sistema automatizado de identificación y susceptibilidad antimicrobiana. En este último punto existe un protocolo de informe de antibiogramas estandarizado según servicio clínico y perfil de susceptibilidad elaborado en conjunto con Infectología. Además existe un sistema de aviso de valores de alerta. En cuanto a documentación existe manual de procedimiento, registro de indicadores y se constata la presencia de control interno de calidad según protocolos autodeterminados. Además, tiene participación en el Programa de Evaluación Externa de la Calidad (PEEC) del Instituto de Salud Pública con resultados aceptables. Para el diagnóstico de $C$. difficile existe disponibilidad ininterrumpida de técnica inmunoenzimática desde el 2007 , sin cambios en el reactivo utilizado y sin restricción de acceso al examen pudiendo ser indicado para cualquier paciente en todos los servicios, en horario diurno, de lunes a domingo.

Prevención-Control de IAAS y acreditación en el Programa de Prevención de Infecciones Intrahospitalarias. Existe una Unidad de IAAS integrada por dos enfermeras competentes contratadas con jornada completa. También participa un médico epidemiólogo con 6 horas semanales asignadas. Sin embargo, casi por 2 años (2009 a julio de 2011), esta Unidad no tuvo profesionales de enfermería o su permanencia fue breve por renuncias sucesivas lo que hizo desaparecer la línea de trabajo ejecutada previamente. Los dos cargos actuales se concretaron en julio y noviembre de 2011, respectivamente. En estos meses, este grupo ha debido no sólo atender la contingencia originada por el brote de infecciones por $C$. difficile sino que también empoderarse del cargo, reorganizar y actualizar normativas y la vigilancia epidemiológica habitual. Por ello no existe información reciente sobre supervisión estructurada respecto al porcentaje de oportunidad y calidad de la higiene de manos o el cumplimiento adecuado en la 
atención de pacientes bajo precauciones de contacto. A fines del año 2006 se hizo la última visita de acreditación por parte del MINSAL para evaluar el Programa local de Prevención y Control de Infecciones Intrahospitalarias. El HUAP obtuvo acreditación destacada. El año 2009 estaba programada una nueva visita que no se pudo efectuar por la pandemia de influenza. La falta de una Unidad de IAAS hasta épocas recientes explica la ausencia prolongada de cursos de prevención y control de IAAS dirigidos al estamento médico.

Programa control de antimicrobianos e información sobre el consumo de estos compuestos. Desde hace más de una década existe un programa de control de antimicrobianos con normativa escrita y actualizada que incluye patología infecciosa común y profilaxis antimicrobiana en Cirugía. El programa está basado en la visita que realiza el médico infectólogo a los pacientes según las recetas diarias recibidas en farmacia para compuestos restringidos, los que deben ser autorizados por este profesional antes de su entrega. La visita era efectiva para suspender antimicrobianos según lo atestigua el porcentaje de interrupciones de tratamiento observadas en el mes de febrero del 2012 que alcanzó >30\% de las indicaciones de la Unidad de Intermedio. Sin embargo, sólo se contaba con un cargo de 44 horas de infectólogo, que no permitía tener cobertura sobre los tratamientos iniciados para el Servicio de Urgencia que no eran trasladados posteriormente a las salas del HUAP y tampoco para responder toda la demanda interna de interconsultas de la subespecialidad. A raíz del brote, se efectuó un análisis del consumo de antimicrobianos desde el año 2009 al 2011, demostrándose en este enfoque que el consumo de cefalosporinas de tercera generación, clindamicina y fluorquinolonas aumentó entre 2009 y 2011.

Empresa de aseo. El aseo, limpieza y desinfección descansa en forma mixta entre una empresa externa que está a cargo de los baños, pasillos y el aseo terminal y en los auxiliares de aseo del HUAP que están a cargo de la limpieza de la unidad clínica y la limpieza de chatas y receptáculos de orina. En el contrato respectivo se menciona el derecho a supervisión que mantiene el HUAP sobre la empresa y que existe un delegado con tal cometido que debe enviar informes quincenales sobre la situación.

\section{Epidemiología de las infecciones por C. difficile en el HUAP}

Descripción epidemiológica. Se tiene conocimiento de la existencia de cuadros de diarrea por $C$. difficile en el HUAP desde el año 2007 a raíz de un brote con 20 casos y por ello se incorporó en forma permanente el test diagnóstico en deposiciones. El año 2008, hubo otro brote y posteriormente cifras endémicas de casos aunque sin un indicador epidemiológico óptimo, porque hasta junio de 2011, no se discriminaba si un paciente con examen positivo representaba un cuadro inicial, una recaída, un test solicitado en fase de convalecencia o una posible inclusión de casos adquiridos fuera del HUAP, lo que puede generar un exceso de casos sobre el valor real. Teniendo en cuenta estos sesgos operativos hasta junio de 2011, los análisis internos demuestran un aumento en la tasa de exámenes positivos de 4,2 el año 2008 a 4,7 el 2009 y luego 9 casos por 10.000 días-paciente el 2010 . El año 2011 la tasa fue de 4,8 por 10.000 días-paciente durante el primer semestre y subió a 16,9 casos nuevos por 10.000 días-paciente el segundo semestre. La tasa vuelve a aumentar en marzo del 2012 (no se obtuvo densidad por 10.000 días-camas ocupadas para este mes). Estas cifras traducen la presencia de un nuevo brote a partir de julio de 2011. Este brote afecta en forma transversal a la institución, no habiendo prácticamente áreas protegidas. Sin embargo, la mayor densidad de casos se concentra en el Servicio de Medicina y UCI. La mitad de los pacientes afectados tiene más de 67 años. Este brote fue notificado directamente al MINSAL y al SEREMI de Salud.

Características clínicas y letalidad. Hasta ahora no se ha explorado en profundidad el perfil clínico que ha tenido este brote y sólo hay datos preliminares de su letalidad. El problema ha sido dedicar recursos humanos (RRHH) al análisis de cada uno de los casos para describir sus características clínicas, algún indicador de gravedad, respuesta o fracaso al tratamiento y consignar los decesos como causa atribuible, asociada o no relacionada a la infección por $C$. difficile. Estos datos son muy importantes para evaluar la posibilidad de que una variante más virulenta de $C$. difficile esté asociada al brote. La revisión de las fichas clínicas de algunos pacientes fallecidos dejó en claro para el grupo visitante de la SOCHINF, que una fracción de los pacientes mencionados en los datos transferidos a los medios de comunicación (cerca de 36 muertes acumuladas desde julio del 2011 a marzo del 2012), correspondieron a pacientes que llegaron infectados al HUAP desde otros centros y fallecieron en este lugar o a casos donde la infección no provocó directamente la muerte. De esta manera, la cifra estimada de letalidad atribuible no puede ser definida con claridad aún y las estimaciones iniciales la sitúan entre 15 y $20 \%$ para el período enero 2011-abril 2012, pero deben ser sometidas a un estudio más profundo.

\section{Factores potenciales involucrados}

Para tener una comprensión acabada de esta situación epidemiológica es necesario describir el problema desde una triple perspectiva: la del hospedero o paciente, del 
ambiente o atención hospitalaria y finalmente del agente. Esta mirada es necesaria para evitar interpretaciones reduccionistas y para entender que la prevención de estos brotes requiere de intervenciones en múltiples frentes.

\section{Factores del hospedero o paciente}

Ya se ha descrito en una sección precedente, que el tipo de pacientes hospitalizados en el HUAP es de una naturaleza frágil debido a las patologías que lo afectan y de mayor gravedad, por lo tanto concentra los factores de riesgo asociados a la infección por $C$. difficile. La mortalidad promedio del HUAP es cercana o equivalente a la de pacientes hospitalizados en salas de tipo intermedio, lo que revela la gravedad intrínseca de ellos. Este tipo de pacientes hace propicio que la colonización por $C$. difficile termine en enfermedad.

\section{Factores ambientales-asistenciales}

Varios factores de naturaleza ambiental y asistencial han contribuido al desarrollo de infecciones por $C$. difficile en el HUAP. Estos factores facilitan la diseminación del agente en el ambiente o su persistencia y la colonización de nuevos pacientes. Sin orden jerárquico se comentan a continuación:

Hacinamiento y ausencia de concepto de unidad clínica. El HUAP fue construido hace muchas décadas, antes que los conceptos de prevención de IAAS estuvieran plenamente desarrollados con la recomendación de minimizar la aglomeración de pacientes. La mayor parte del HUAP tiene salas de hospitalización con 8 camas en cada una de ellas con distancias pequeñas entre cada paciente hecho que facilita la transmisión cruzada. Un avance se produjo el año 2006 con el retiro de camillas de pacientes hospitalizados desde los pasillos de estos sectores. Otro hecho relevante es la ausencia del concepto de unidad clínica, que considera a cada paciente y su entorno inmediato como un sector separado del resto de los pacientes, que debe ser limpiado cada día y donde el instrumental médico debe ser dedicado en forma exclusiva o desinfectado antes de ser usado en otro paciente.

Higiene de manos y cultura de prevención de IAAS. No se dispuso de información oficial sobre el porcentaje del personal de salud que adhiere a higiene de manos antes de atender a cada paciente pero informaciones verbales sitúan esta cifra en $40 \%$ aproximadamente. Aunque no se ha medido en el último tiempo, el grado de cumplimiento a las normas de prevención de IAAS es bajo entre el personal según las entrevistas efectuadas por esta comisión a pesar de los esfuerzos de capacitación.

Restricción de insumos para salas con precauciones de contacto. Antes de las medidas adoptadas en abril de 2012, los insumos necesarios para los pacientes bajo precauciones de contacto tales como delantales y guantes, estaban supeditados a un presupuesto fijo para las diferentes unidades clínicas, que no era flexible ante cambios epidemiológicos. Ello generaba restricciones para contar con estos insumos y un nivel de precaución subóptimo que puede facilitar la transmisión de microorganismos.

Ausencia de Unidad IAAS efectiva y capacitación personal de salud. La ausencia de profesionales de enfermería a cargo de esta unidad en forma efectiva desde el año 2009 hasta noviembre del 2011 facilitó el problema debido a la falta de un equipo humano que mantuviera vigilancia, supervisión y acciones correctivas sobre el mismo. Tampoco es posible que este equipo pudiera hacer intervenciones mayores desde el primer día de estar constituido en plenitud hacia fines del año 2011, debido al período necesario que se requiere para ejecutar las funciones del cargo y restablecer el liderazgo. Además, las horas asignadas del médico epidemiólogo (6 horas semanales) son claramente insuficientes para poder analizar en forma apropiada la vasta tarea de un hospital complejo y hacer los análisis oportunos sobre el brote, los que como se demostró, aún requieren dedicación. De la misma manera, la ausencia de un médico infectólogo con mayor dedicación a esta Unidad, debido a la subdotación de este recurso y a su absorción por el programa de control de antimicrobianos, contribuye al problema al restar recursos humanos con potencial para capacitar al resto del equipo de salud en la normativa de prevención y/o analizar con mayor profundidad el devenir del brote y sus consecuencias. Finalmente, la capacitación formal en prevención y control de IAAS ha sido escasa entre el personal de salud, especialmente entre el estamento médico y ha sido focalizada por la contingencia sólo hacia el tema puntual de $C$. difficile pero no hacia toda la problemática de las IAAS. Esto ha contribuido a que no exista en los últimos años una clara cultura de prevención de este riesgo en la atención de pacientes.

Dotación de enfermeros supervisores. Existe un escaso número de enfermeros supervisores que pueda corregir las transgresiones en el cumplimiento en las normas de prevención en IAAS y que además cumplen numerosas tareas. Ello permite que no se modifique la cultura de trabajo y que se mantenga el riesgo de transmisión.

Auxiliares de aseo y limpieza de equipos médicos. El aseo de chatas y recipientes para la recolección de orina de los pacientes está a cargo de auxiliares de servicio que cumplen numerosas otras tareas y que deben atender a un gran volumen de pacientes. El aseo de estos equipos se realiza en forma manual, con bajo nivel de supervisión y 
con posibilidades de errores en su limpieza y desinfección. Estos factores contribuyen a la persistencia del agente en el ambiente y equipos médicos. La automatización en el aseo de estos equipos sólo está disponible en la sala de cohorte para los infectados en el sexto piso y desde hace unas dos semanas, lo que ha permitido una mejor racionalización en las tareas que cumplen los auxiliares de aseo pero sólo en ese lugar.

Control sobre el consumo de antimicrobianos y dotación de médicos infectólogos. Los RRHH dedicados al control de antimicrobianos son insuficientes a pesar de su labor diaria y las altas tasas de interrupción de tratamientos observados, lo que claramente no ha podido frenar la expansión en el consumo de estos compuestos observados en los últimos años, especialmente de aquellas moléculas que aparecen más ligadas al riesgo de adquirir esta enfermedad. Esta subdotación ha facilitado que el gatillante más relevante en la génesis de este problema, el uso de antimicrobianos, haya incrementado su peso en forma progresiva en los últimos años. Además, no hay control efectivo sobre los antimicrobianos indicados en el Servicio de Urgencia, donde varios pacientes permanecen hospitalizados hasta su traslado interno o externo.

Desinfección ambiental. La desinfección ambiental adecuada, incluyendo la de la unidad clínica, la de chatas y receptáculos de orinas y la puertas, barandas y pasillos, depende de que exista un número suficiente de auxiliares de aseo para esta labor o funcionarios de la empresa de aseo contratada para tal servicio, que tengan una capacitación adecuada y que sea supervisada. Como se ha señalado, la dotación de los auxiliares de aseo del HUAP no es apropiada a no ser que se incorporen medidas para automatizar parte de su labor. La capacitación en prevención de IAAS ha sido incidental por la contingencia del brote de infecciones por $C$. difficile pero no estructural o de amplia cobertura. Por otra parte, el nivel de preparación de los funcionarios de la empresa externa para desarrollar el aseo terminal o de áreas clínicas es bajo y además tienen una alta rotación que limita cualquier esfuerzo de capacitación progresiva. Todos estos factores facilitan la persistencia y diseminación de este y otros agentes y se agravan por la ausencia de roles supervisores suficientes entre el personal clínico y ante las labores de la empresa externa de aseo. Por otra parte, se debe mencionar que las bases del contrato con la empresa externa consideraban el uso de cloro como desinfectante sólo para baños, lavamanos y duchas pero no en un formato flexible según la situación epidemiológica. Asimismo, el desinfectante maestro considerado en estas bases era el amonio cuaternario pero no se mencionaba la generación mínima necesaria que debían tener estos productos, los que en caso de pertenecer a generaciones más antiguas, no tienen la capacidad de control sobre varios agentes infecciosos en presencia de suciedad. El rol supervisor por parte del HUAP sobre esta empresa recayó en el Jefe de Lavandería, perteneciente al área administrativa y no clínica, que debido a la demanda generada por su propio trabajo y a la naturaleza de su perfil profesional, le impidió ejecutar una supervisión según el espíritu del contrato. Las evaluaciones efectuadas por el Jefe de Calidad del HUAP han demostrado un cumplimiento insuficiente y la experiencia con la empresa de aseo externa actual ha sido mal evaluada, motivando una nueva licitación en curso.

\section{Factores del agente}

Clostridium difficile es una bacteria grampositiva anaerobia, productora de esporas responsable de un porcentaje importante de diarreas asociadas a antimicrobianos y casos graves de colitis pseudomembranosa y megacolon tóxico. Desde el año 2000 se ha reconocido en varios países un aumento de la incidencia de la enfermedad apareciendo información de brotes nosocomiales y de una nueva cepa (BI/NAP1/027) con características de hipervirulencia. En esta cepa se ha descrito la producción de una toxina binaria de significado clínico incierto y la presencia de una deleción de 18 pares de bases en el gen tcdC, que es un regulador de la producción de toxinas A y B, pero cuya relación con una hiperproducción de toxinas no está del todo aclarado. También se ha descrito una deleción puntual en la posición 117 del mismo gen que genera una proteína truncada que podría asociarse a una mayor síntesis de toxinas.

En el Instituto de Salud Pública entre agosto 2011 y abril 2012 se han recibido muestras procedentes desde el HUAP de pacientes con diarrea por $C$. difficile y que tenían previamente un resultado positivo para toxina A/B por técnica inmunoenzimática. Para este período se cuenta con información de 42 cepas (30 completamente caracterizadas) en las que se han identificado por electroforesis de campo pulsado (PFGE) 9 tipos genéticos. En ellos, el denominado tipo Cl-Cdf-Sma001 (14 casos) se caracteriza por presentar la deleción de 18 pares bases en el gen tcdC, lo que no se ha encontrado en los otros grupos clonales. Sin embargo, este grupo clonal no corresponde al clon BI/NAP1/027 descrito en otros países, pero sí a probable variante de este.

Los resultados globales a la fecha indican la presencia de un brote policlonal con un tipo genético predominante (Cl-Cdf-Sma001; 47\% de los estudiados) que es una posible variante del tipo genético NAP1. Sin embargo, no es posible concluir que nos encontramos frente a un fenómeno nuevo ya que no contamos con datos previos a agosto 2011, ni tampoco afirmar que es exclusivo del HUAP ya que no tenemos información de otros hospitales nacionales. No existe aún comprobación de que se trate 
de una cepa hipervirulenta ni más transmisible, aunque podría estar relacionada a cepas con este comportamiento en brotes extranjeros.

Es recomendable continuar el estudio para buscar otros factores tales como deleciones puntuales en el gen regulador en la posición 117 y presencia de toxina binaria. Además, es necesario realizar estudios que demuestren una hiperproducción de toxinas en estas cepas u otros análisis que permitan inferir una mayor duración en el ambiente o mayor transmisibilidad que ayude a explicar la aparición del brote.

Cabe mencionar que el personal médico de la institución refiere que desde la aparición del brote, la evolución de los pacientes afectados ha sido de un manejo más difícil, resolución más lenta del cuadro y mayor número de complicaciones, sin que esto se haya objetivado. Por lo anterior, es recomendable continuar con la caracterización clínica del brote para evaluar si los pacientes con infecciones por el grupo Cl-Cdf-Sma001 han tenido un cuadro más grave en cuanto a complicaciones y letalidad versus aquellos pacientes infectados con otras cepas así como también si ha habido mayor gravedad global de los pacientes del brote versus un comparador histórico.

\section{Medidas implementadas}

En reunión con la Unidad de IAAS y en visitas a los servicios de Medicina, Cirugía y UCI, se pudo constatar la implementación de las siguientes medidas:

Definición de unidad de paciente: a partir de julio 2011 se estableció la definición clara de la unidad de cada paciente, incluyendo en ella el velador y los insumos utilizados en forma individual. La implementación completa de la medida se logró en abril 2012 en el área de cohorte centralizada. La UCI cuenta con una clara definición de unidad de paciente demarcada con línea roja que facilita el cumplimiento de medidas.

Aislamiento en cohorte: esta medida se implementó en dos etapas:

1. Aislamiento en cohorte de casos aislados de C. difficile en salas de los distintos servicios clínicos desde julio 2011.

2. Cohorte centralizada: medida implementada a partir del 5 de abril de 2012. Incluye el aislamiento de los casos de $C$. difficile en salas de 6 pacientes en el Servicio de Medicina, sector hombres sexto piso. Esta medida significó reducir el número de camas disponibles de 8 a 6 por sala, aumentado el espacio físico entre los pacientes. Los casos se ubican en salas diferenciando casos sintomáticos con toxina $C$. difficile positiva (casos activos), casos en resolución o asintomáticos con toxina $C$. difficile positiva (casos inactivos) y pacientes que hayan estado en contacto con casos (contactos), cada grupo en salas independientes. Además se limitó el acceso de personal al sector de aislamiento, excluyendo a los alumnos de pre y postgrado de estas salas y dejando personal exclusivo (enfermeras, técnicos paramédicos y auxiliares de servicio) para atender estos pacientes. Los pacientes deben permanecer en sus salas y utilizan sus chatas en cada unidad con limitado acceso al baño. La ficha médica y los registros de enfermería se mantienen fuera de las salas.

Higiene de manos con agua y jabón: desde marzo de 2012 se suspendió en la cohorte centralizada el uso de alcohol-gel en la atención de pacientes con $C$. difficile, fomentado el lavado de manos con agua y jabón. En visita se constató la presencia de varios carteles difundiendo la medida. El equipo de IAAS no realizó supervisión directa de esta práctica hasta el mes de abril y sólo se pudo disponer de una supervisión externa realizada por EU Marlene Valenzuela (IAAS Servicio Metropolitano Central) que da cuenta de una adherencia global a lavado de manos de alrededor de $40 \%$ a nivel hospitalario (informe verbal).

Precaución de contacto: medida implementada desde julio 2011. Incluye el uso de delantal de manga larga, plástico, desechable y guantes en la atención de todos los casos de $C$. difficile. La implementación completa de esta medida se logró desde principios de abril 2012; desde esa fecha se cuenta con un número suficiente y estable de insumos en todos los turnos. La implementación de esta medida significó aumentar la frecuencia del retiro de desechos cada 4 horas, debido a la mayor utilización de insumos.

Desinfección ambiental: en forma habitual en HUAP la desinfección ambiental es realizada utilizando amonios cuaternarios. Desde junio 2011 se estableció como medida el realizar desinfección ambiental tanto para aseos concurrentes como terminales con cloro al 1\%. Sin embargo, en la implementación de esta medida hubo dificultades, dado que la dilución era realizada sin protocolos claros por la empresa externa de aseo y la duración de los productos podía ser prolongada, incluso hasta 15 días. Desde abril 2012, con la incorporación de la cohorte centralizada, se implementó la compra directa de cloro por cada servicio clínico con dilución protocolizada por auxiliares de servicio bajo capacitación directa de enfermeras de IAAS.

Lavado de chatas en lavadora automática: desde la semana del 23 de abril 2012 se implementó el lavado automático de chatas en lavadora en la cohorte centralizada del Servicio de Medicina hombres. 
Restricción del uso de clindamicina y levofloxacina en el Servicio de Urgencia: el 20 de abril de 2012 se restringió el uso de clindamicina y levofloxacina en pacientes con neumonía aspirativa atendidos en el Servicio de Urgencia reemplazando la cobertura anti anaerobios por el uso de metronidazol.

Capacitaciones de personal: Para el estamento médico sólo se han realizado las siguientes capacitaciones incidentales relacionadas al brote de $C$. difficile: Una en el servicio de Cirugía, dos en el Servicio de Medicina y una en la UCI relacionada al uso de antimicrobianos. Para el personal de salud de otros estamentos se han realizado capacitaciones anuales para el personal no médico, tanto profesional como técnico, pero con muy baja cobertura. El año 2011 se realizaron tres capacitaciones específicas en $C$. difficile.

\section{Impacto de las medidas implementadas}

Luego de la incorporación e implementación de todas las medidas descritas a partir de la primera semana de abril, se ha observado una reducción de la incidencia de casos.

\section{Recomendaciones}

Disminuir el hacinamiento hospitalario en forma local y mediante la pronta apertura de nuevos hospitales públicos en la Región Metropolitana. En forma transitoria, mantener la cohorte centralizada con 6 camas por salas y posteriormente reducir todas las salas a este nuevo estándar de camas.

Fortalecer las actividades de supervisión a través de un mayor número de RRHH dedicados a evaluar las medidas de prevención de IAAS, tales como adherencia a la higiene de manos, precauciones de contacto, aseo y desinfección de la unidad clínica y de los equipos e instrumental de la unidad, incluyendo la supervisión sobre los procesos de desinfección ambiental.

Automatizar, tal como ya sucede en la cohorte, el proceso de aseo de chatas y receptáculos urinarios, para homogeneizar la calidad del proceso y disminuir la carga de trabajo de los auxiliares de servicio.

Aumentar el número de baños y duchas para pacientes en las salas de hospitalización de Medicina y Cirugía.

Fortalecer el programa de control de antimicrobianos y evaluación infectológica de pacientes a través de una dotación efectiva de médicos infectólogos, estimada en al menos dos cargos de jornada completa, con incorporación de parte de sus actividades a labores dentro de la Unidad de IAAS. El restablecimiento de las labores del médico infectólogo debe efectuarse a la brevedad con el fin de apoyar el adecuado manejo de los casos y un mejor control del brote.

Incorporar un médico microbiólogo o un especialista en Laboratorio Clínico con mención en Microbiología, al menos en jornada parcial, al Laboratorio Central del HUAP e incorporar en forma rutinaria el estudio de agentes virales respiratorios para pacientes hospitalizados que lo ameriten, con el propósito de contribuir al uso racional de antimicrobianos y disminuir su consumo.

Fortalecer las funciones de la Unidad de IAAS a través de un tercer cargo de enfermera integrante, participación de médicos infectólogos, microbiológo y expansión en las horas contratadas del médico epidemiólogo.

Incorporar en forma estable y estructurada, amplios programas de capacitación en prevención y control de IAAS, en todos los estamentos y especialmente entre los médicos para fortalecer una cultura apropiada de calidad y seguridad asistencial. En estos programas deben incluirse alumnos de pre y postgrado asignados al HUAP.

Profundizar el estudio del brote a través de una mayor caracterización del perfil clínico de los pacientes afectados, incluyendo la letalidad atribuible y evaluar mediante estudios de diseño adecuado un posible comportamiento más agresivo del agente involucrado incluyendo la respuesta al tratamiento. Es necesario también, ampliar la caracterización microbiológica para confirmar o descartar la hipótesis de hipertoxigenicidad y mayor transmisibilidad.

Los contratos con las empresas externas de aseo y limpieza deben ser más exigentes respecto a la capacitación mínima que sus recursos humanos deberían tener en materias hospitalarias y considerar una supervisión efectiva por parte del hospital contratista sobre estas labores.

\section{Comentarios finales}

El brote de infecciones por $C$. difficile ha sucedido en otras instituciones, ha afectado al HUAP y seguirá emergiendo en otros lugares. La conjunción de pacientes debilitados, hacinamiento hospitalario, deficiencias en los procesos de atención del paciente incluyendo el consumo excesivo de antimicrobianos y la contratación de empresas externas de aseo y limpieza son hechos comunes en el sistema público de salud en el país. Este escenario transversal es propicio para la aparición de brotes por agentes infecciosos nosocomiales y no se reduce a $C$. difficile sino que incluye el problema de Enterococcus spp resistente a vancomicina y otras bacterias resistentes.

Es necesario por lo tanto entender que las soluciones para estos problemas no pueden ser reduccionistas o simplistas y que su judicialización no las resuelve. Por el contrario, la experiencia acumulada por el equipo 
local y las limitaciones encontradas para avanzar en el control del brote, deben ser traspasadas a otros equipos de salud del país para estar mejor preparados ante esta $\mathrm{u}$ otras contingencias similares. En esa perspectiva, la SOCHINF ha participado en esta evaluación y asesoría de este problema. Esto ha permitido enriquecer el conocimiento nacional sobre la materia y avanzar en lograr un mejor estándar de salud para lo población.

En la visita efectuada al HUAP, la comisión evaluadora pudo constatar el compromiso de la autoridad, de la
Unidad de IAAS así como de numerosas otras personas y equipos, para resolver este problema epidemiológico y además, quedó de manifiesto que las medidas adoptadas han resultado ser efectivas para reducir el brote. El duro aprendizaje que ha vivido el HUAP debe ser aprovechado no sólo por sus propios actores, sino que también por el resto del país y los avances alcanzados, deben seguir siendo apoyados.

Santiago, mayo del 2012 